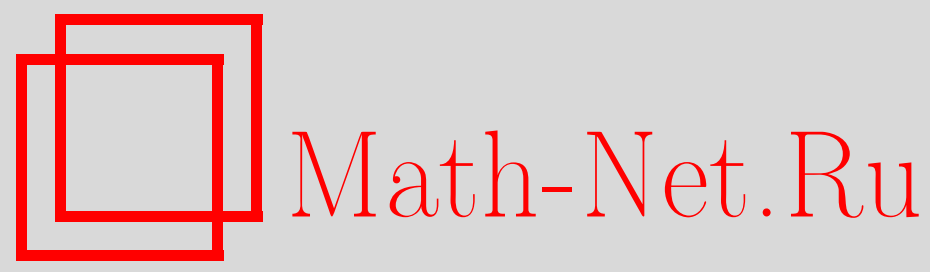

М. И. Дьяченко, $U$-сходимость рядов Фурье с монотонными и с положительными коэффициентами, Матем. заметки, 2001, том 70, выпуск 3, 356-365

DOI: https://doi.org/10.4213/mzm748

Использование Общероссийского математического портала Math-Net.Ru подразумевает, что вы прочитали и согласны с пользовательским соглашением http://www.mathnet.ru/rus/agreement

Параметры загрузки:

IP : 54.80 .73 .141

26 апреля 2023 г., 15:39:01

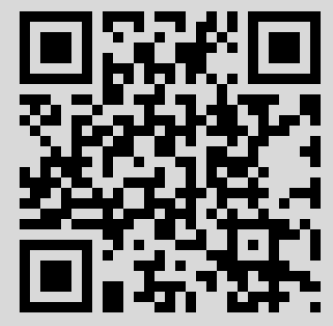




\section{U-СХОДИМОСТЬ РЯДОВ ФУРЬЕ С МОНОТОННЫМИ И С ПОЛОЖИТЕЛЬНЫМИ КОЭФФИЦИЕНТАМИ}

\section{М.И. Дьяченко}

В статье рассматриваются вопросы $u$-сходимости кратных рядов Фурье с монотонньми и с положительными коэффициентами в метриках $L_{p}$.

Библиограбоия: 12 названий.

1. Введение. Пусть натуральное $m \geqslant 2, T=[-\pi, \pi), \mathbb{Z}^{m}$-множество всех целочисленных точек в $\mathbb{R}^{m}, 2 \pi$-периодическая по каждому переменному функция $f(\boldsymbol{x}) \in L\left(T^{m}\right)$ и

$$
\sum_{n \in \mathbb{Z}^{m}} a_{\boldsymbol{n}}(f) e^{i \boldsymbol{n} \boldsymbol{x}}
$$

- ее ряд Фурье, где

$$
a_{\boldsymbol{n}}(f)=\frac{1}{(2 \pi)^{m}} \int_{T^{m}} f(\boldsymbol{t}) e^{-i \boldsymbol{n} \boldsymbol{t}} d \boldsymbol{t}
$$

и $\boldsymbol{n} \boldsymbol{x}=n_{1} x_{1}+n_{2} x_{2}+\cdots+n_{m} x_{m}$. Хорошо известно, что в многомерном случае существует много различных определений сходимости ряда (1). Например, прямоугольные частичные суммы определяются формулой

$$
S_{\boldsymbol{N}}(f ; \boldsymbol{x})=\sum_{\left|k_{1}\right| \leqslant N_{1}} \cdots \sum_{\left|k_{m}\right| \leqslant N_{m}} a_{\boldsymbol{k}}(f) e^{i \boldsymbol{k} \boldsymbol{x}},
$$

где $\boldsymbol{N} \in \mathbb{Z}^{m} \cap[0,+\infty)^{m}$ (если $\boldsymbol{N}=(r, \ldots, r)$, мы будем назьвать такие суммы кубическими) и сферические

$$
S_{R}(f ; \boldsymbol{x})=\sum_{|\boldsymbol{k}| \leqslant R} a_{\boldsymbol{k}}(f) e^{i \boldsymbol{k} \boldsymbol{x}},
$$

где $R \geqslant 1$. Соответственно, если существуют

$$
\lim _{\min _{1 \leqslant j \leqslant m} N_{j} \rightarrow \infty} S_{\boldsymbol{N}}(f ; \boldsymbol{x})=\alpha, \quad \lim _{r \rightarrow \infty} S_{(r, \ldots, r)}(f ; \boldsymbol{x})=\alpha, \quad \lim _{R \rightarrow \infty} S_{R}(f ; \boldsymbol{x})=\alpha,
$$

то скажем, что ряд (1) сходится в точке $\boldsymbol{x} \kappa$ числу а по прямоугольникам (по Прингсхейму), по кубам и по сферам. Аналогичные определения можно дать и для сходимости в пространствах $L_{p}\left(T^{m}\right)$.

Результаты данной статьи получены при финансовой поддержке Российского фонда фундаментальных исследований, гранты № 00-01-00042 и № 99-01-00062. 
Заметим, что в вопросах сходимости прямоугольные и сферические частичные суммы ведут себя совершенно различньп образом. Существуют и другие способы определения сходимости кратных рядов, такие как сходимость по полиэдрам, по гиперболическим крестам и т.д. Поэтому представляется интересньм дать определение сходимости, включающее все описанные выше. Отметим, что во всех определениях сходимости множества, по которым берутся суммы, симметричны относительно всех координатных гиперплоскостей и содержат, вместе с каждой точкой, целочисленный параллелепипед с вершинами в данной точке и в начале координат. Представляется уместным дать следующие определения.

ОПРЕДЕЛЕНИЕ 1. Пусть ограниченное множество $U \subset \mathbb{Z}^{m}$. Тогда скажем, что $U \in A$, если из того, что $k \in U$, следует, что

$$
\prod_{j=1}^{m}\left[-\left|k_{j}\right|,\left|k_{j}\right|\right] \cap \mathbb{Z}^{m} \subseteq U
$$

ОПРЕДЕЛЕНИЕ 2. Скажем, что числовой ряд

$$
\sum_{n \in \mathbb{Z}^{m}} c_{n}
$$

$u$-сходится к числу $\alpha$, если для любого $\varepsilon>0$ существует число $M$ такое, что для любого $U \in A$, для которого $\left\{\boldsymbol{n} \in \mathbb{Z}^{m}:|\boldsymbol{n}| \leqslant M\right\} \subseteq U$, имеем

$$
\left|S_{U}-\alpha\right| \equiv\left|\sum_{n \in U} c_{n}-\alpha\right|<\varepsilon
$$

Очевидно, что если ряд $u$-сходится, то он сходится по Прингсхейму, по сферам, по гиперболическим крестам и т.д. Определение такой сходимости было впервые использовано $\Phi . Г$. Арутюняном [1], [2] в статьях о представлении функций многих переменных кратньми тригонометрическими рядами.

Некоторые задачи $u$-сходимости рядов Фурье в метриках пространств $L_{p}[3]$, почти всюду [4] и всюду [5] были ранее рассмотрены автором.

Упомянем здесь основной результат работы [3]. Вначале приведем определение классов С. М. Никольского.

ОПРЕДЕЛЕНИЕ 3 . Если $k$ - натуральное число, то $k$-я $р$ азность функции $f$ в точке $\boldsymbol{x}$ с шагом $\boldsymbol{t}$ это

$$
\Delta_{k}(f, \boldsymbol{x}, \boldsymbol{t})=\sum_{r=0}^{k}(-1)^{r} C_{k}^{r} f(\boldsymbol{x}+r \boldsymbol{t})
$$

а если $1 \leqslant p \leqslant \infty$ и $f(\boldsymbol{x}) \in L_{p}\left(T^{m}\right)$, то модуль гладкости порядка $k$ в пространстве $L_{p}\left(T^{m}\right)$ это

$$
\omega_{k}(f, \delta)_{p}=\sup _{\boldsymbol{t} \in \mathbb{R}^{m}:|\boldsymbol{t}| \leqslant \delta}\left\|\Delta_{k}(f, \boldsymbol{x}, \boldsymbol{t})\right\|_{p}, \quad \text { где }|\boldsymbol{t}|=\sqrt{t_{1}^{2}+\cdots+t_{m}^{2}} .
$$


ОПРЕДЕЛЕНИЕ 4 . Пусть $1 \leqslant p \leqslant \infty$ и $\alpha>0$. Тогда обозначим через $H_{p}^{\alpha}\left(T^{m}\right)=$ $\left\{f(\boldsymbol{x}) \in L_{p}\left(T^{m}\right):\right.$ для всех $k>\alpha$ имеем $\omega_{k}(f, \delta)_{p}=O\left(\delta^{\alpha}\right)$ при $\left.\delta \rightarrow+0\right\}$. Если здесь заменить $O$ на $o$, то получившийся класс обозначим $h_{p}^{\alpha}\left(T^{m}\right)$.

Теорема А. Если $m \geqslant 2,1 \leqslant p \leqslant \infty, p \neq 2$ и функиия $f(\boldsymbol{x}) \in h_{p}^{(m-1)|1 / 2-1 / p|}\left(T^{m}\right)$, то ряд Фурье функиии $f(\boldsymbol{x})$ и-сходится в метрике $L_{p}\left(T^{m}\right)$.

ТЕОремА Б. Если $m \geqslant 2 u 1 \leqslant p \leqslant \infty, p \neq 2$, то существует такая функиия $f(\boldsymbol{x}) \in H_{p}^{(m-1)|1 / 2-1 / p|}\left(T^{m}\right)$, что ее ряд Фурье и-расходится в метрике $L_{p}\left(T^{m}\right)$. Кроме того, при $p=\infty$ ряд Фурье в соответствующем примере и-расходится всюду.

Из этих результатов следует, что нужна достаточно большая (степенная) гладкость функции $f(\boldsymbol{x}) \in L_{p}, p \neq 2$, для $u$-сходимости ее ряда Фурье в соответствующей метрике. Здесь естественно возникает вопрос: можем ли мы заменить количественные требования в теореме А на качественные? Например, мы можем рассматривать эту задачу для кусочно-монотонных функций, для функций с монотонными или с неотрицательными коэффициентами Фурье. В данной статье мы изучим два последних случая.

Разумеется, вначале мы должны дать определение тригонометрического ряда с монотонньми коэффициентами. В многомерном случае имеется несколько различных определений. Наиболее естественным является следующее.

ОПРЕДЕЛЕНИЕ 5 . Пусть $\boldsymbol{n}, \boldsymbol{k} \in \mathbb{Z}^{m}$. Тогда скажем, что $\boldsymbol{k} \geqslant \boldsymbol{n}$, если $k_{j} \geqslant n_{j}$ при $j=1,2, \ldots, m$. Далее, пусть задана числовая последовательность $\left\{a_{\boldsymbol{n}}\right\}_{\boldsymbol{n} \in \mathbb{Z}^{m}, \boldsymbol{n} \geqslant \mathbf{1}}$ (здесь через 1 обозначен вектор, все координаты которого равны 1). Мы назовем ее монотонной, если из того, что $\boldsymbol{k} \geqslant \boldsymbol{n}$, вытекает, что $0 \leqslant a_{\boldsymbol{k}} \leqslant a_{\boldsymbol{n}}$.

Заметим, что по теореме М. Рисса если $1<p<\infty$ и функция $f(\boldsymbol{x}) \in L_{p}\left(T^{m}\right)$, то для любого вектора $\boldsymbol{g}=\left(g_{1}, \ldots, g_{m}\right)$ с $\left|g_{j}\right|=1$ при $j=1,2, \ldots, m$ ряд

$$
\sum_{\substack{n \in \mathbb{Z}^{m}: \\ \operatorname{sign} n_{j}=g_{j}, j=1,2, \ldots, m}} a_{\boldsymbol{n}}(f) e^{i \boldsymbol{n} \boldsymbol{x}}
$$

также является рядом Фурье некоторой функции из пространства $L_{p}\left(T^{m}\right)$. Поэтому мы будем изучать ряды Фурье вида

$$
\sum_{\boldsymbol{n} \in \mathbb{Z}^{m}: \boldsymbol{n} \geqslant \mathbf{1}} a_{\boldsymbol{n}}(f) e^{i \boldsymbol{n} \boldsymbol{x}}
$$

с монотонными коэффициентами.

Ниже через $C$ будем обозначать абсолютные положительные постоянные, не обязательно равные в различных случаях, через $C(p)$ положительные постоянные, зависящие только от $p$, и т.д.

В статье [6] автор установил следующий результат.

Теорема В. Пусть $2<p<\infty$, функиия $f(\boldsymbol{x}) \in L_{p}\left(T^{m}\right)$ и имеет ряд Фурье (2) с монотонными коэффициентами. Тогда

$$
J_{p}(f) \equiv\left(\sum_{n \in \mathbb{Z}^{m}: \boldsymbol{n} \geqslant \mathbf{1}}\left|a_{\boldsymbol{n}}(f)\right|^{p}\left(\prod_{j=1}^{m} n_{j}\right)^{p-2}\right)^{1 / p} \leqslant C(p, m)\|f\|_{L_{p}} .
$$


Отметим, что при $1<p \leqslant 2$ аналогичная теорема содержится в хорошо известной теореме Харди-Литлвуда.

С другой стороны, в [7] автор установил такой результат.

Теорема Г. Пусть $p \in(2 m /(m+1), 2)$ и мы имеем кратный тригонометрический ряд

$$
\sum_{\boldsymbol{n} \in \mathbb{Z}^{m}: \boldsymbol{n} \geqslant \mathbf{1}} a_{\boldsymbol{n}} e^{i \boldsymbol{n} \boldsymbol{x}}
$$

с монотонными коэффициентами такой, что

$$
J_{p} \equiv\left(\sum_{n \in \mathbb{Z}^{m}: \boldsymbol{n} \geqslant \mathbf{1}}\left|a_{\boldsymbol{n}}\right|^{p}\left(\prod_{j=1}^{m} n_{j}\right)^{p-2}\right)^{\frac{1}{p}}<\infty .
$$

Тогда этот рядявяяется рядом Фурье некоторой функиии $f(\boldsymbol{x}) \in L_{p}\left(T^{m}\right) u\|f\|_{L_{p}} \leqslant$ $C(p, m) J_{p}$.

Следующее утверждение будет выведено из теорем В и Г в п. 2.

Теорема 1. Если $m \geqslant 2,2 m /(m+1)<p<\infty, p \neq 2$, функиия $f(\boldsymbol{x}) \in L_{p}\left(T^{m}\right) u$ имеет ряд Фурье вида (2) с монотонными коэффициентами, то этот ряд и-сходится $\kappa f(\boldsymbol{x})$ в метрике $L_{p}$.

В том же пункте мы докажем следующую теорему.

Теорема 2. Если $m \geqslant 2$ и $1<p \leqslant 2 m /(m+1)$, то существует функиия $f(\boldsymbol{x}) \in$ $L_{p}\left(T^{m}\right)$, ряд Фурье которой имеет вид (2) с монотонными коэффичиентами, но и-расходится в метрике $L_{p}$.

В п. 3 будут доказаны следующие результаты для функций с неотрицательными коэффициентами Фурье.

Теорема 3. Пусть $m \geqslant 2, p=2 k$, әде $k=2,3, \ldots$, а функиия $f(\boldsymbol{x}) \in L_{p}\left(T^{m}\right)$ и имеет неотрицательные коэффициенты Фурье. Тогда ряд Фурье функиии $f(\boldsymbol{x})$ u-сходится к ней в метрике $L_{p}$.

ТеОрема 4. Пусть $m \geqslant 2 u p \in(1, \infty)$ не является четным иелым числом. Тогда существует функиия $f(\boldsymbol{x}) \in L_{p}\left(T^{m}\right)$ с неотрицательными коэффициентами Фурье такая, что ее ряд Фурье и-расходится в метрике $L_{p}$.

2. $U$-сходимость рядов Фурье с монотонными коэффициентами. Вначале докажем теорему 1. По теореме А имеем

$$
\left(\sum_{n \in \mathbb{Z}^{m}: \boldsymbol{n} \geqslant \mathbf{1},}\left(a_{\boldsymbol{n}}(f)\right)^{p}\left(\prod_{j=1}^{m} n_{j}\right)^{p-2}\right)^{1 / p}<\infty .
$$

Поэтому существует последовательность положительных чисел $\left\{\alpha_{\boldsymbol{n}}\right\}_{\boldsymbol{n}} \geqslant \mathbf{1}$ такая, что последовательности $\left\{\alpha_{\boldsymbol{n}} a_{\boldsymbol{n}}\right\}_{\boldsymbol{n}} \geqslant \mathbf{1}$ и $\Lambda \equiv\left\{1 / \alpha_{\boldsymbol{n}}\right\}_{\boldsymbol{n} \geqslant \mathbf{1}}$ монотонны и

$$
C_{0}=\left(\sum_{\boldsymbol{n} \in \mathbb{Z}^{m}: \boldsymbol{n} \geqslant \mathbf{1},}\left(\alpha_{\boldsymbol{n}} a_{\boldsymbol{n}}(f)\right)^{p}\left(\prod_{j=1}^{m} n_{j}\right)^{p-2}\right)^{1 / p}<\infty .
$$


Пусть $\left\{d_{\nu}\right\}_{\nu=1}^{\infty}$, где $\nu=\nu(\boldsymbol{n})$, это последовательность $\Lambda$, занумерованная в порядке невозрастания, и

$$
U_{r}=\left\{\boldsymbol{n} \in \mathbb{Z}^{m} \cap[1, \infty)^{m}: \nu(\boldsymbol{n}) \leqslant r\right\}
$$

при $r=1,2, \ldots$ Поскольку последовательность $\Lambda$ монотонна, очевидно, что $U_{r} \in A$ для любого $r$. Теперь определим функцию

$$
\varphi(\boldsymbol{x})=\sum_{r=1}^{\infty}\left(\sum_{\boldsymbol{n} \in U_{r}} \alpha_{\boldsymbol{n}} a_{\boldsymbol{n}} e^{i \boldsymbol{n} \boldsymbol{x}}\right) \cdot\left(d_{r}-d_{r+1}\right) .
$$

Из (3) и из теоремы Б следует, что ряд (4) сходится в метрике $L_{p}$. Легко проверить, что $\varphi(\boldsymbol{x})=f(\boldsymbol{x})$, поскольку они имеют одинаковые коэффициенты Фурье. Аналогично, для заданного $U \in A$ мы можем представить соответствующую частичную сумму как

$$
S_{U}(f ; \boldsymbol{x})=\sum_{r=1}^{|U|}\left(\sum_{\boldsymbol{n} \in U_{r}^{\prime}} \alpha_{\boldsymbol{n}} a_{\boldsymbol{n}} e^{i \boldsymbol{n} \boldsymbol{x}}\right) \cdot\left(d_{r}^{\prime}-d_{r+1}^{\prime}\right),
$$

где $d_{|U|+1}^{\prime}=0, d_{r}^{\prime}=d_{r}$ и $U_{r}^{\prime}=U_{r}$ при $1 \leqslant r \leqslant r_{0}, r_{0}$ зависит от $N(U)=\max \left\{N:[1, N]^{m}\right.$ $\subseteq U\}$ и $r_{0} \rightarrow \infty$ при $N(U) \rightarrow \infty$. Итак, имеем (см. (3)-(5))

$$
\begin{aligned}
\left\|S_{U}(f ; \boldsymbol{x})-\varphi(\boldsymbol{x})\right\|_{L_{p}} & \leqslant \sum_{r=r_{0}+1}^{\infty} C(p, m) C_{0}\left(d_{r}-d_{r+1}\right)+\sum_{r=r_{0}+1}^{|U|} C(p, m) C_{0}\left(d_{r}^{\prime}-d_{r+1}^{\prime}\right) \\
& \leqslant 2 C(p, m) C_{0} d_{r_{0}} \rightarrow 0
\end{aligned}
$$

при $N(U) \rightarrow \infty$. Утверждение теоремы 1 следует из этой оценки.

Теперь перейдем к доказательству теоремы 2 . Пусть задано $m \geqslant 2$. Фиксируем некоторое $\alpha \in(0,(m-1) /(2 m)]$ и определим функцию одного переменного

$$
g_{\alpha}(t)=\frac{1}{2}+\sum_{k=0}^{\infty} \frac{1}{2 n_{k}^{\alpha}\left(\ln n_{k}\right)^{1 /(2 m)}} \sum_{l=n_{k-1}+1}^{n_{k}} e^{i l t},
$$

где $n_{k}=2^{2^{k}}$ при $k=0,1,2, \ldots$ и $n_{-1}=0$. Ряд (6) имеет монотонные коэффициенты, поэтому он сходится на $(0,2 \pi)$. Заметим, что при любом $p \in(1,1 /(1-\alpha)]$ имеем

$$
\begin{aligned}
\left(\int_{T}\left|g_{\alpha}(t)\right|^{p} d t\right)^{1 / p} & \leqslant C+\sum_{k=0}^{\infty} \frac{1}{2 n_{k}^{\alpha}\left(\ln n_{k}\right)^{1 /(2 m)}}\left(\int_{T}\left|\sum_{l=n_{k-1}+1}^{n_{k}} e^{i l t}\right|^{p} d t\right)^{1 / p} \\
& \leqslant C\left(1+\sum_{k=0}^{\infty} \frac{n_{k}^{1-1 / p}}{2 n_{k}^{\alpha}\left(\ln n_{k}\right)^{1 /(2 m)}}\right) \\
& \leqslant C\left(1+\sum_{k=0}^{\infty} \frac{1}{2^{k /(2 m)}}\right)<\infty
\end{aligned}
$$

Пусть теперь у нас некоторое $p \in(1,2 m /(m+1)]$. Возьмем $\alpha=1-1 / p$ и определим функцию

$$
f(\boldsymbol{x})=g_{\alpha}\left(x_{1}\right) g_{\alpha}\left(x_{2}\right) \cdots g_{\alpha}\left(x_{m}\right)
$$


Согласно (7) функция $f(x) \in L_{p}\left(T^{m}\right)$. Далее, поскольку коэффициенты Фурье любой функции $g_{\alpha}\left(x_{j}\right), j=1,2, \ldots, m$, монотонно убьвают, коэффициенты Фурье функции $f(\boldsymbol{x})$ монотонны. Теперь докажем, что ряд Фурье функции $f(\boldsymbol{x}) u$-расходится в метрике $L_{p}$.

Вначале определим две последовательности целочисленных множеств $\left\{U_{k}\right\}_{k=2}^{\infty}$ и $\left\{V_{k}\right\}_{k=2}^{\infty}$ из класса $A$. Пусть

$$
U_{k}=\left(\bigcup_{j=1}^{m}\left(\prod_{r=1}^{j-1}\left[1, n_{k}\right]\right) \times\left[1, n_{k-1}\right] \times\left(\prod_{r=j+1}^{m}\left[1, n_{k}\right]\right)\right) \cap \mathbb{Z}^{m}
$$

и

$$
\begin{array}{r}
V_{k}=U_{k} \cup\left\{\boldsymbol{n} \in \mathbb{Z}^{m}: n_{j}>n_{k-1} \text { при } j=1,2, \ldots, m\right. \\
\text { и } \left.\left|\boldsymbol{n}-\left(n_{k-1}, \ldots, n_{k-1}\right)\right| \leqslant n_{k}-n_{k-1}\right\}
\end{array}
$$

при $k=2,3, \ldots$. Ясно, что все эти множества принадлежат классу $A$. Далее, имеем

$$
\left\|S_{V_{k}}(f ; \boldsymbol{x})-S_{U_{k}}(f ; \boldsymbol{x})\right\|_{L_{p}}=\left(\frac{1}{2 n_{k}^{\alpha}\left(\ln n_{k}\right)^{1 /(2 m)}}\right)^{m}\left\|\sum_{\substack{\boldsymbol{l} \in \mathbb{Z}^{m}: \boldsymbol{l} \geqslant \mathbf{1} \\ 1 \leqslant|\boldsymbol{l}| \leqslant n_{k}-n_{k-1}}} e^{i \boldsymbol{l} \boldsymbol{x}}\right\|_{L_{p}} .
$$

Заметим, что справедливы следующие оценки снизу для норм ядер Дирихле:

$$
\begin{array}{r}
\left\|\sum_{\substack{\boldsymbol{l} \in \mathbb{Z}^{m}: \boldsymbol{l} \geqslant \mathbf{1} \\
1 \leqslant|\boldsymbol{l}| \leqslant n_{k}-n_{k-1}}} e^{i \boldsymbol{l \boldsymbol { x }}}\right\|_{L_{p}} \\
\geqslant C(p) \cdot \begin{cases}\left(n_{k}-n_{k-1}\right)^{(m-1) / 2} & \text { при } p<\frac{2 m}{m+1}, \\
\left(n_{k}-n_{k-1}\right)^{(m-1) / 2}\left(\ln \left(n_{k}-n_{k-1}\right)\right)^{1 / p} & \text { при } p=\frac{2 m}{m+1} .\end{cases}
\end{array}
$$

Первая из этих оценок легко выводится из хорошо известного результата В. А. Ильина [8], а вторая - из соответствующей оценки В. А. Юдина [9].

Поскольку $n_{k}-n_{k-1} \geqslant n_{k} / 2$ при $k \geqslant 2$, из (8) следует, что для $p=2 m /(m+1)$ имеем

$$
\left\|S_{V_{k}}(f ; \boldsymbol{x})-S_{U_{k}}(f ; \boldsymbol{x})\right\|_{L_{p}} \geqslant C \cdot \frac{n_{k}^{(m-1) / 2} \cdot\left(\ln n_{k}\right)^{1 / p}}{n_{k}^{m \alpha} \cdot\left(\ln n_{k}\right)^{1 / 2}}=C(p) \cdot\left(\ln n_{k}\right)^{1 / p-1 / 2} \rightarrow \infty
$$

при $k \rightarrow \infty$. Если $1<p<2 m /(m+1)$, то

$$
\begin{aligned}
\left\|S_{V_{k}}(f ; \boldsymbol{x})-S_{U_{k}}(f ; \boldsymbol{x})\right\|_{L_{p}} & \geqslant C(p) \cdot \frac{n_{k}^{(m-1) / 2}}{n_{k}^{m \alpha} \cdot\left(\ln n_{k}\right)^{1 / 2}} \\
& \geqslant C(p) \cdot\left(n_{k}\right)^{((m-1) / 2+m / p-m) / 2} \rightarrow \infty
\end{aligned}
$$

при $k \rightarrow \infty$.

Итак, теорема 2 полностью доказана. 
3. $U$-сходимость рядов Фурье с неотрицательными коэффициентами. Вначале мы докажем теорему 3 . Пусть $p=2 q$, где натуральное $q \geqslant 2$, и пусть мы имеем полином с неотрицательными коэффициентами

$$
P_{\nu}(\boldsymbol{x})=\sum_{\boldsymbol{n} \in \mathbb{Z}^{m}:-\nu \leqslant n_{j} \leqslant \nu} a_{\boldsymbol{n}} e^{i \boldsymbol{n} \boldsymbol{x}} .
$$

Тогда

$$
\begin{aligned}
\left\|P_{\nu}(\boldsymbol{x})\right\|_{L_{p}}^{p} & =\left\|P_{\nu}^{q}(\boldsymbol{x})\right\|_{L_{2}}=\sum_{\boldsymbol{n} \in \mathbb{Z}^{m}}\left|a_{\boldsymbol{n}}\left(P_{\nu}^{q}\right)\right|^{2} \\
& =\sum_{\boldsymbol{n} \in \mathbb{Z}^{m}}\left(\sum_{\substack{\boldsymbol{k}(1)+\cdots+\boldsymbol{k}(q)=n \\
-\nu \leqslant \boldsymbol{k}(l) j \leqslant \nu \\
1 \leqslant l \leqslant q, 1 \leqslant j \leqslant m}} a_{\boldsymbol{k}(1)} \ldots a_{\boldsymbol{k}(q)}\right)^{2} \equiv \sum_{\boldsymbol{n} \in \mathbb{Z}^{m}} \sigma_{\boldsymbol{n}, \nu}^{2} .
\end{aligned}
$$

Пусть функция $f(\boldsymbol{x}) \in L_{p}\left(T^{m}\right)$ и имеет неотрицательные коэффициенты Фурье. Положим $P_{\nu}(\boldsymbol{x})=S_{(\nu, \ldots, \nu)}(f ; \boldsymbol{x})$ при $\nu=1,2, \ldots$ Тогда по теореме М. Рисса имеем $P_{\nu}(\boldsymbol{x}) \rightarrow f(\boldsymbol{x})$ при $\nu \rightarrow \infty$ в метрике $L_{p}$. Поскольку для любого $\boldsymbol{\nu} \in \mathbb{Z}^{m}$ неотрицательные величины

$$
\sigma_{\boldsymbol{n}, \nu \uparrow} \sum_{\boldsymbol{k}(1)+\cdots+\boldsymbol{k}(q)=\boldsymbol{n}} a_{\boldsymbol{k}(1)} \cdots a_{\boldsymbol{k}(q)} \equiv \sigma_{\boldsymbol{n}}
$$

при $\nu \rightarrow \infty$, по теореме Фату последовательность $\left\{\sigma_{n}\right\}_{n \in \mathbb{Z}^{m}} \in l_{2}$. В частности, $\sigma_{\boldsymbol{n}}<\infty$ для любого $\boldsymbol{n}$.

Теперь, если задано некоторое $\varepsilon>0$, то выберем $N$ так, что

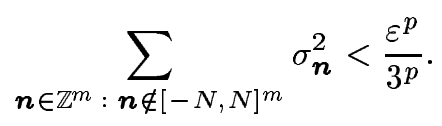

Далее, фиксируем $N$ и выберем $\nu$ так, что

$$
\left|\sigma_{n, \nu}-\sigma_{n}\right|^{2}<\frac{\varepsilon^{p}}{3^{p} \cdot 2^{m} \cdot N^{m}}
$$

для каждого $\boldsymbol{n} \in[-N, N]^{m}$.

Наконец, если задано множество $U \in A$ такое, что $U \supset[-\nu, \nu]^{m}$, то найдем натуральное $N_{1} \geqslant N$ такое, что $U \subset\left[-N_{1}, N_{1}\right]^{m}$ и

$$
\left\|f(\boldsymbol{x})-S_{\left(N_{1}, \ldots, N_{1}\right)}(f ; \boldsymbol{x})\right\|_{L_{p}}<\frac{\varepsilon}{3} \text {. }
$$

Таким образом (см. (9)-(12)), имеем

$$
\begin{aligned}
& \left\|f(\boldsymbol{x})-S_{U}(f ; \boldsymbol{x})\right\|_{L_{p}} \\
& \quad \leqslant\left\|f(\boldsymbol{x})-S_{\left(N_{1}, \ldots, N_{1}\right)}(f ; \boldsymbol{x})\right\|_{L_{p}}+\left\|S_{\left(N_{1}, \ldots, N_{1}\right)}(f ; \boldsymbol{x})-S_{U}(f ; \boldsymbol{x})\right\|_{L_{p}} \\
& \quad \leqslant \frac{\varepsilon}{3}+\left(\sum_{\boldsymbol{n} \in[-N, N]^{m}}\left|\sigma_{\boldsymbol{n}}-\sigma_{\boldsymbol{n}, \nu}\right|^{2}\right)^{1 / p}+\left(\sum_{\boldsymbol{n} \in \mathbb{Z}^{m} \backslash[-N, N]^{m}} \sigma_{\boldsymbol{n}}^{2}\right)^{1 / p}<\varepsilon
\end{aligned}
$$

а это и требовалось доказать.

Заметим, что достаточно доказать теорему 4 в двумерном случае. Нам понадобится следующий вспомогательньй результат. 
ПРЕДЛОЖЕНИЕ 1. Пусть $p \in(1, \infty)$ и для любого натурального $N$ существуют одномерный полином

$$
P_{N}(x)=\sum_{k=0}^{r(N)} c_{k}(N) e^{i k x}
$$

әде $c_{k}(N) \geqslant 0$ при $k=0,1, \ldots, r(N)$, и числа $\left\{\delta_{k}(N)\right\}_{k=0}^{r(N)}$, где $\delta_{k}(N)=0$ или $\delta_{k}(N)=1$ при всех $k$, такие, что если полином

$$
Q_{N}(x)=\sum_{k=0}^{r(N)} \delta_{k}(N) c_{k}(N) e^{i k x}
$$

то $\left\|Q_{N}(x)\right\|_{L_{p}(T)}>N\left\|P_{N}(x)\right\|_{L_{p}(T)}$. Тогда существует такая функиия $f\left(x_{1}, x_{2}\right) \in$ $L_{p}\left(T^{2}\right)$ с неотрицательными коэффициентами Фурье, что ее ряд Фурье и-расходится в метрике $L_{p}$.

ДокАЗАТЕЛЬСТво. Не ограничивая общности, мы можем предположить, что последовательность $\left\{r\left(2^{l}\right)\right\}_{l=1}^{\infty}$ строго монотонна. Пусть $\alpha_{l}=\left\|P_{2^{l}}(x)\right\|_{L_{p}(T)}$ и функция

$$
f\left(x_{1}, x_{2}\right)=\sum_{l=1}^{\infty} \frac{1}{l^{2} \alpha_{l}} \sum_{k=0}^{r\left(2^{l}\right)} c_{k}\left(2^{l}\right) e^{i k x_{1}} e^{i\left(r\left(2^{l}\right)-k\right) x_{2}}=\sum_{l=1}^{\infty} \frac{1}{l^{2} \alpha_{l}} R_{l}\left(x_{1}, x_{2}\right)
$$

Поскольку

$$
\left\|R_{l}\left(x_{1}, x_{2}\right)\right\|_{L_{p}\left(T^{2}\right)} \leqslant C\left\|P_{2^{l}}(t)\right\|_{L_{p}(T)}=C \alpha_{l},
$$

ряд (13) сходится в метрике $L_{p}$.

Теперь определим две последовательности множеств $\left\{U_{l}\right\}_{l=1}^{\infty}$ и $\left\{V_{l}\right\}_{l=1}^{\infty}$ из класса $A$. Пусть

$$
U_{l}=\left\{\left(n_{1}, n_{2}\right) \in \mathbb{Z}^{2}:\left|n_{1}\right|+\left|n_{2}\right| \leqslant r\left(2^{l}\right)\right\}
$$

и

$$
V_{l}=U_{l} \backslash\left\{\left(n_{1}, n_{2}\right) \in \mathbb{Z}^{2}:\left|n_{1}\right|+\left|n_{2}\right|=r\left(2^{l}\right) \text { и } \delta_{\left|n_{1}\right|}\left(2^{l}\right)=1\right\}
$$

при $l=1,2, \ldots$.

Тогда при $l=1,2, \ldots$ имеем

$$
\begin{aligned}
\left\|S_{U_{l}}\left(f ;\left(x_{1}, x_{2}\right)\right)-S_{V_{l}}\left(f ;\left(x_{1}, x_{2}\right)\right)\right\|_{L_{p}\left(T^{2}\right)} & =\frac{1}{l^{2} \alpha_{l}}\left\|\sum_{k=0}^{r\left(2^{l}\right)} \delta_{k}\left(2^{l}\right) c_{k}\left(2^{l}\right) e^{i k\left(x_{1}-x_{2}\right)}\right\|_{L_{p}\left(T^{2}\right)} \\
& >\frac{1}{l^{2} \alpha_{l}}\left\|Q_{2^{l}}(t)\right\|_{L_{p}(T)}>\frac{2^{l}}{l^{2}} \rightarrow \infty
\end{aligned}
$$

при $l \rightarrow \infty$. Итак, предложение 1 полностью доказано.

В статье [10] Г. Шапиро (см., также [11]) доказал, что для любого $p \in(1, \infty)$, которое не является четным целым числом, существуют одномерные полиномы

$$
P^{*}(x)=\sum_{k=0}^{k_{0}} c_{k}^{*} e^{i k x}
$$


с $c_{k}^{*} \geqslant 0$ при $k=0,1, \ldots, k_{0}$ и

$$
Q^{*}(x)=\sum_{k=0}^{k_{0}} \delta_{k}^{*} c_{k}^{*} e^{i k x}
$$

где $\delta_{k}^{*}= \pm 1$ для всех $k$, такие, что $\left\|Q^{*}(x)\right\|_{L_{p}(T)}>\left\|P^{*}(x)\right\|_{L_{p}(T)}$.

Теперь докажем, что при указанном выше $p$ для любого $N$ существуют полиномы

$$
P_{N}^{*}(x)=\sum_{k=0}^{r(N)} c_{k}^{*}(N) e^{i k x}
$$

с $c_{k}^{*}(N) \geqslant 0$ при $k=0,1, \ldots, k_{0}$ и

$$
Q_{N}^{*}(x)=\sum_{k=0}^{r(N)} \delta_{k}^{*}(N) c_{k}^{*}(N) e^{i k x}
$$

где $\delta_{k}^{*}(N)= \pm 1$ для всех $k$, такие, что

$$
\left\|Q_{N}^{*}(x)\right\|_{L_{p}(T)}>(2 N+1)\left\|P_{N}^{*}(x)\right\|_{L_{p}(T)} .
$$

Следующее доказательство этого факта было дано С. В. Конягиным. Мы публикуем его здесь с любезного согласия Конягина.

Нам понадобится следующее утверждение, являющееся частным случаем известной леммы Фейера (см. [12, с. 77]).

Лемма. Пусть $p \in(1, \infty)$ и $P(x)$ - одномерный тригонометрический полином. Тогда для любого $\varepsilon>0$ существует число $M_{0}$ такое, что для любого натурального $M>M_{0}$ имеем

$$
\left.\left|\left(\frac{1}{\sqrt{2 \pi}} \int_{T}|P(x)|^{p} d x\right)^{2}-\int_{T}\right| P(x) P(M x)\right|^{p} d x \mid<\varepsilon .
$$

Пусть $\left\|Q^{*}(x)\right\|_{L_{p}(T)}>q\left\|P^{*}(x)\right\|_{L_{p}(T)}$, где $q>1$. Возьмем $\alpha>0$ таким, что

$$
\left\|Q^{*}(x)\right\|_{L_{p}(T)}>(q+\alpha)\left\|P^{*}(x)\right\|_{L_{p}(T)} .
$$

Затем, используя лемму, выберем натуральное $M>\operatorname{deg} P^{*}(x)$ таким, что

$$
\begin{aligned}
\left\|Q^{*}(x) Q^{*}(M x)\right\|_{L_{p}(T)} & >\left(1-\frac{\alpha}{q+\alpha}\right) \frac{1}{(2 \pi)^{1 /(2 p)}}\left\|Q^{*}(x)\right\|_{L_{p}(T)}^{2} \\
& >\frac{1}{(2 \pi)^{1 /(2 p)}} q(q+\alpha)\left\|P^{*}(x)\right\|_{L_{p}(T)}^{2}>q^{2}\left\|P^{*}(x) P^{*}(M x)\right\|_{L_{p}(T)} .
\end{aligned}
$$

Заметим, что поскольку $M>\operatorname{deg} P^{*}(x)$, любой коэффициент полинома $Q^{*}(x) \times$ $Q^{*}(M x)$ равен соответствующему коэффищиенту полинома $P^{*}(x) P^{*}(M x)$, умноженному либо на 1 , либо на -1 . При этом все коэффициенты полинома $P^{*}(x) P^{*}(M x)$ неотрицательны. Таким образом, построены полиномы

$$
P^{* *}(x)=P^{*}(x) P^{*}(M x)=\sum_{k=0}^{k_{1}} c_{k}^{* *} e^{i k x}
$$


с $c_{k}^{* *} \geqslant 0$ при $k=0,1, \ldots, k_{1}$ и

$$
Q^{* *}(x)=Q^{*}(x) Q^{*}(M x)=\sum_{k=0}^{k_{1}} \delta_{k}^{* *} c_{k}^{* *} e^{i k x}
$$

где $\delta_{k}^{* *}= \pm 1$ для всех $k$, такие, что

$$
\left\|Q^{* *}(x)\right\|_{L_{p}(T)}>q^{2}\left\|P^{* *}(x)\right\|_{L_{p}(T)} .
$$

Повторяя этот процесс, выводим требуемое утверждение.

$$
\begin{aligned}
& \text { Пусть } P_{N}(x)=2 P_{N}^{*}(x) \text { и } Q_{N}(x)=Q_{N}^{*}(x)+P_{N}^{*}(x) . \text { Поскольку } \\
& \qquad \begin{aligned}
\left\|Q_{N}(x)\right\|_{L_{p}(T)} & \geqslant\left\|Q_{N}^{*}(x)\right\|_{L_{p}(T)}-\left\|P_{N}^{*}(x)\right\|_{L_{p}(T)} \\
& >2 N\left\|P_{N}^{*}(x)\right\|_{L_{p}(T)}=N\left\|P_{N}(x)\right\|_{L_{p}(T)},
\end{aligned}
\end{aligned}
$$

эта пара полиномов удовлетворяет условию предложения 1. Итак, доказательство теоремы 4 завершено.

Автор выражает глубокую признательность члену-корреспонденту РАН Б. С. Кашину, профессору С. В. Конягину и профессору В. А. Юдину за полезные обсуждения.

\section{СПИСОК ЦИТИРОВАННОЙ ЛИТЕРАТУРЫ}

[1] Арутюнян Ф. Г. Представление функций кратными рядами // Докл. АН АрмССР. 1977. T. 64 . № 2. C. $72-76$.

[2] Арутюнян Ф.Г. Представление измеримых функций многих переменных кратными тригонометрическими рядами // Матем. сб. 1985. Т. 126. №2. С. 267-285.

[3] Дьяченко М. И. U-сходимость кратных рядов Фурье // Изв. РАН. Сер. матем. 1995. Т. 59. № 2. C. $128-142$.

[4] Дьяченко М.И. U-сходимость почти всюду двойных рядов Фурье // Матем. сб. 1995. T. 186. №1. C. 47-64.

[5] Дьяченко М. И. Двумерные классы Ватермана и $u$-сходимость рядов Фурье // Матем. сб. 1999. T. 190. № 7. C. 23-40.

[6] Dyachenko M. I. Multiple trigonometric series with lexicographically monotone coefficients // Anal. Math. 1990. V. 16. № 3. P. 173-190.

[7] Дьяченко М. И. Нормы ядер Дирихле и некоторых других тригонометрических полиномов в пространствах $L_{p} / /$ Матем. сб. 1993. Т. 184. № 3. С. 3-20.

[8] Ильин В. А. Проблемы локализации и сходимости для рядов Фурье по фундаментальным системам функций оператора Лапласа // УМН. 1968. Т. 23. № 2. С. 60-120.

[9] Юдин В.А. Докторская диссертация. М.: МИАН им. В. А. Стеклова, 1991.

[10] Shapiro H.S. Majorant problems for Fourier coefficients // Quart. J. Math. 1975. V. 26. № 101. P. 9-18.

[11] Boas R. P. Majorant problems for trigonometric series // J. d'Analyse Math. 1962/63. V. 10. P. 253-271.

[12] Бари Н. К. Тригонометрические ряды. М.: Физматгиз, 1961. 